\title{
Lines in the Sand: An Anthropological Discourse on Wildlife Tourism
}

\author{
Georgette Leah Burns ${ }^{1}$
}

Received: 21/08/2010

${ }^{1}$ Griffith University, Queensland, Australia; Phone: 61737353649 , e-mail: Leah.Burns@griffith.edu.au

Supervisors: Assoc. Professor Jim Macbeth and Assoc. Professor Susan Moore

Institution awarding the Ph. D. Degree: Murdoch University, Western Australia

Date of defence: 16/09/2009

(c) 2010 International University College. All rights reserved

Citation: Burns, G.L. (2010) Lines in the Sand: An Anthropological Discourse on Wildlife Tourism. Doctoral dissertation summary. European Journal of Tourism Research 3(2), pp. 119-122

\section{Goal and objectives of the dissertation Goal}

The management of wildlife tourism has been dominated by ideologies informed by western colonialism and its values of nature. These ideologies, made transparent through communicative and interpretative discourses, influence the way management policies and practices are devised and enacted. The inherent scientific and utilitarian views are supported by a doctrine of separation. This is apparent in the dualism posed, and enacted, between nature and culture that sees humans as being the sole carriers of culture that separates them from the uncultured and uncivilised world of nature into which all other animals, and certainly untamed wildlife, belong. It justifies the use of non-humans for human purposes and continues to allow us to treat non-human animals and other forms of nature in often abominable ways.

This thesis explores one of the central dilemmas facing wildlife tourism: what are the implications of the separation constructed between wildlife and people in wildlife tourism settings? This exploration is an interdisciplinary journey drawing on understandings, methods and theories from anthropology, tourism and environmental science. It promotes an engagement between anthropology and the scholarly discourses of tourism and environmental science, and a broad approach to defining and understanding the wildlife tourism community.

\section{Objectives}

Approaches to wildlife tourism management are needed which will optimise stakeholder satisfaction with wildlife tourism, within the constraints of ensuring sustainability of the wildlife product. This thesis is designed around a series of objectives aimed at understanding these constraints and finding new approaches. Guiding the research that informs this thesis are questions that ask why management of the human-wildlife interface occurs in the way it does, and what values underpin the constructions of wildlife that, in turn, enable and legitimise the management. 


\section{Methodology}

This thesis investigates two situations in which wildlife tourism occurs in Australia. Fraser Island and Penguin Island are two wildlife tourism destinations on opposite sides of the continent with very different wildlife but some very similar issues. From these two contexts data was collected through interviews, focus groups, participant observation, and from literary and documentary sources. Understanding the empirical data collected from these case studies is facilitated through a social constructionist view of discourse analysis that allows an unpacking of the messages and a stance from which to challenge the dominant ideologies that frame management and interaction.

\section{Results}

The case studies and theories explored in this thesis highlight the way wildlife tourism is managed. The dominant constructions underlying management policies and practices are exposed, and challenged, and alternatives posed.

I demonstrate throughout that anthropology, in its incarnation as environmental anthropology and as a team player in a necessarily interdisciplinary approach to understanding and resolving environmental issues, has much to offer. This engagement has the potential to enhance not only the sustainable future of nature-based activities like wildlife tourism but also the relevance of anthropology in the postcolonial contemporary world. Thus the thesis contributes to the body of literature on the anthropology of tourism, environmental anthropology and wildlife tourism.

\section{Theoretical conclusions}

The need for a holistic framework encompassing all the stakeholders in any wildlife tourism venture is proposed. This approach to wildlife tourism is best serviced by examining perspectives, values and concerns of all members of the wildlife tourism community at any given destination. It is only through this type of holistic and situated focus that we can hope to effectively understand, and then manage, in the best interests of all parties.

The argument is made for a rethinking of the way wildlife tourism interactions are managed in some settings. The ideology of separation, enacted both conceptually and physically to create maintain boundaries, is demonstrated through the two case studies and the ways in which interactions between humans and wildlife are currently managed. An alternative is posed, that by reconstructing management in settings where wildlife tourists may be more accepting of their own responsibility towards nature, a model can be developed that allows people and wildlife to co-exist without 'killing' the natural instincts of either.

\section{Practical application of the dissertation}

The thesis proposes ways of reconstructing the management of wildlife tourism that have practical applications for wildlife tourism managers.

The application of an anthropological lens to the study of tourism and environment, particularly to the subfield of wildlife tourism, has some sound benefits for enhancing understanding and assessing management practices and implications. For wildlife tourism scholars, researchers, planners and managers the significance of this thesis lies in its contribution to a greater understanding of the wildlife tourism community, as well as its exposure of the dominant ideologies governing management and the challenges they pose for alternative ways forward. An important outcome from this research is the insights it provides into how wildlife-based tourism in Australia can continue in a sustainable framework that is suitable for the wildlife, the tourists, other stakeholders, and the managers of this complex phenomenon.

\section{Content of the dissertation}

Abstract of chapter one

Chapter One provides an overview of the thesis, explaining the aim of the research and discussing its significance. Because an understanding of terminology is important, some of the key terms used throughout the thesis are introduced in this first chapter 
which concludes with an outline of, and rationale for, the structure of the thesis.

\begin{abstract}
of chapter two
The two case studies, from which empirical evidence was collected for this thesis, are introduced in Chapter Two. Fraser Island, off the Queensland coast, and Penguin Island, off the Western Australian coast, are both destinations for wildlife tourism. This chapter discusses the location of these two case studies, the wildlife they contain, and their wildlife tourism community (managers, tourists, and other stakeholders).
\end{abstract}

\section{Abstract of chapter three}

Chapter Three explains the rationale of the design behind collection methods and how data were collected during the research. Topics covered include the combined research strategies of ethnography and case studies, as well as a description of each of the data collection methods used (sampling, interviews, literature and documentary sources, and participant observation). The ethical considerations pertinent to the collection of data and how they were dealt with are also discussed here. Finally, the chapter turns to an explanation of the data analysis and theorisation. Discourse analysis is presented as a guiding analytical and theoretical tool for collecting, collating and understanding the data. An overview of social constructionism explains how this perspective is used as a context for the study and understanding of nature and the environment in this thesis.

\section{Abstract of chapter four}

In Chapter Four I discuss where, and why, data from the case studies fits into bodies of literature on anthropology of tourism and environmental anthropology and why this literature provides both a sound, and unique, framework for the thesis. This includes exploration of the historical relationship between anthropology and tourism. The history of environmental anthropology is also discussed, along with its recent rise and importance in Australia. Opportunities and challenges for increasing both the academic and applied engagement of anthropology with the field of environmental science are outlined.

\begin{abstract}
of chapter five
Chapter Five discusses literature on the contested notion of community as it is relevant to wildlife tourism. The wildlife tourism community referred to throughout the thesis is made up of many stakeholder groups and individuals with interests in wildlife tourism that are often diverse and conflicting, and the importance of inclusion of these stakeholders in decisions about wildlife tourism is made implicit. Some of the issues addressed include involvement of stakeholders, community attitudes and values, and their impact on people, wildlife and the sustainability of tourism. The importance of considering all these issues when planning for, and managing, wildlife tourism, is discussed.
\end{abstract}

\section{Abstract of chapter six}

Chapter Six examines the links between wildlife tourism, conservation and sustainability, arguing that conservation practices are informed by the dominant, Eurocentric, ideology of nature that is a legacy of colonisation. The relationship between nature and culture explored in this chapter provides a framework that is later applied to the separation of wildlife and people in wildlife tourism settings by management authorities. The use, or denial, of anthropomorphism is discussed as an example of a discourse that influences the construction of wildlife tourism and interactions between people and wildlife.

\section{Abstract of chapter seven}

Chapter Seven focuses on the Fraser Island case study as an example of wildlife management in a tourism context. It illustrates the dilemma of deciding between prioritising of management of wildlife for people or management of people for wildlife. The first, and only, human death from a dingo attack on Fraser Island occurred in April 2001, and was immediately followed by a governmentordered cull of dingoes. This chapter explores issues surrounding both this decision and the management strategies implemented afterwards. Based on interviews with a variety of 
stakeholders in the wildlife tourism community, many conflicting perspectives on human-wildlife interaction as a component of tourism are identified. The conclusion is drawn that while strategies for managing dingoes are essential, if such attacks are a consequence of humans feeding wildlife and resultant wildlife habituation, then strategies for successfully managing people are also necessary for this type of wildlife tourism to be sustainable.

\section{Abstract of chapter eight}

Chapter Eight uses a framework of social constructionism to analyse the Fraser Island Dingo Management Strategy (FIDMS). To ensure the validity and effectiveness of wildlife management, it is necessary to consider the assumptions underlying wildlife management and perpetuated through management discourses. This chapter deconstructs dingo management on Fraser Island. It argues that the process of construction in defining environmental problems and possible solutions needs to be recognised and the nature of the ideologies underpinning environmental management acknowledged. Management practices need to be consistent with discourses, reflecting priorities, addressing the problem and facilitating the fulfilment of expectations. Ultimately, entrenched ideologies and assumptions need to be challenged to create new possibilities for action.

\section{Abstract of chapter nine \\ Chapter Nine offers a comparison of the similarities and differences in wildlife tourism on Fraser Island and Penguin Island. This chapter examines the roles of key stake- holders in the wildlife tourism community, focusing on the values placed on wildlife, as well as the nature of human-wildlife interactions and management policies and practices in the two wildlife tourism settings. The relationships between people and dingoes, and between people and penguins, are explored and shown to have shifted over time, and be subject to different controls in each location. Data show that as perceptions}

of wildlife change, management of interactions must also change.

\section{Abstract of chapter ten}

In Chapter Ten the physical and conceptual separation of people and wildlife in the two wildlife tourism settings is explored and its implications interrogated and challenged. The construction of boundaries between people and wildlife are analysed in terms of the ideologies and values that underpin them. The role of anthropomorphism in creating and maintaining these boundaries is investigated and the argument made that anthropomorphising penguins may assist their conservation while the 'anthropodenial' of dingoes hinders it. The chapter calls for a reconstruction of people and wildlife, and a reconstruction of the management of their interactions. Embracing the ideals of ecotourism, and empowering of wildlife tourists to take responsibility for their own actions is suggested as a way forward.

\section{Abstract of chapter eleven}

Chapter Eleven comments on the value and utility of the notion of community for understanding, and ultimately managing sustainably, wildlife tourism. The chapter advocates the need to challenge constructions underpinning wildlife management and the accepted interactions between wildlife and people, arguing for recognition of humans as part of the natural system and a relaxing of management strategies that focus on the separation of people and nature. It also encourages further research in the interdisciplinary overlap between anthropology, environmental science, and tourism: the anthropology of environmental tourism. The combination of perspectives within this area offers crucial insights that can lead to positive outcomes for the environmental and social challenges faced by wildlife, and for the sustainability of wildlife tourism in Australia.

Available: This thesis is available on Murdoch's Open Repository at http://researchrepository.murdoch.edu.au/714 